Research Paper

\title{
Enhancer RNA - P2RY2e induced by estrogen promotes malignant behaviors of bladder cancer
}

\author{
Mengting Ding ${ }^{1,2^{*}}$, Hengji Zhan ${ }^{3 *}$, Xinhui Liao ${ }^{3^{*}}$, Aolin $\mathrm{Li}^{1^{*}}$, Yucheng Zhong 3 , Qunjun Gao ${ }^{3}$, Yuchen Liu ${ }^{3 凶}$, \\ Weiren Huang ${ }^{3 凶}$, Zhiming $\mathrm{Cai}^{3^{\circledR}}$ \\ 1. Clinical Medicine College of Anhui Medical University, Department of Urology, Shenzhen Second People's Hospital, Shenzhen 518000, Guangdong, China. \\ 2. Anhui Medical University, Hefei 230032, Anhui Province, China \\ 3. Department of Urology, Shenzhen Second People's Hospital, the First Affiliated Hospital of Shenzhen University, Shenzhen 518000, China \\ * Equal contributors \\ $\square$ Corresponding author: Yuchen Liu, Weiren Huang, ZhimingCai. E-mail: liuyuchenmdcg@163.com (Yuchen Liu); pony8980@163.com (Weiren Huang); \\ caizhiming2000@163.com (Zhiming Cai) \\ (C) Ivyspring International Publisher. This is an open access article distributed under the terms of the Creative Commons Attribution (CC BY-NC) license \\ (https://creativecommons.org/licenses/by-nc/4.0/). See http://ivyspring.com/terms for full terms and conditions.
}

Received: 2018.05.08; Accepted: 2018.06.23; Published: 2018.07.27

\begin{abstract}
Enhancers are transcriptional regulatory elements that increase target gene expression. It has reported that enhancers could universally transcribe into enhancer RNAs (eRNAs) with stimulation. Increasing evidence showed eRNAs participated in various disease processes including malignant tumors. P2RY2 enhancer RNA (P2RY2e) is an estrogen-responsive eRNA and involved in the development of breast cancer. However, the relationship between P2RY2e and bladder cancer $(B C a)$ is unclear. In the study, we discovered that P2RY2e was upregulated in $B C a$ tissues and estrogen-treated cells. Estrogen promoted the malignant abilities of BCa cells. P2RY2e knockdown by CRISPR-Cas 1 3a inhibit the cell multiplication, invasion and migration. Additionally, the cell apoptosis was facilitated. What's more, downregulation of $\mathrm{P} 2 \mathrm{RY} 2 \mathrm{e}$ could weaken the cancer-promoting effects of estrogen on BCa. Our study revealed that $\mathrm{P} 2 \mathrm{RY} 2 \mathrm{e}$ played a carcinogenic role in $\mathrm{BCa}$ and estrogen might promote the initiation of $\mathrm{BCa}$ by inducing $\mathrm{P} 2 \mathrm{RY} 2 \mathrm{e}$. We provide a potential therapeutic target for $\mathrm{BC}$ a and a new perspective for the tumorigenesis of bladder cancer.
\end{abstract}

Key words: P2RY2e; Estrogen; Bladder cancer; CRISPR-Cas13a

\section{Background}

Estrogen plays significant roles in carcinogenesis, especially in BCa $[1,2]$. Even though BCa mostly occurs in men, female patients present with more invasive phenotype and have lower survival rates [2-6]. Current therapies for $\mathrm{BCa}$, such as transurethral resection or radical cystectomy and intravesical instillation of Bacillus Calmette-Guerin (BCG), do not remarkably improve the overall survival rates of advanced $\mathrm{BCa}[7,8]$. Deficient knowledge regarding molecular mechanisms of this difference in gender incidence is a barrier to the improvement of clinical management of BCa. More exploration of this aspect might open a new prospect for the prevention or treatment for BCa.

Purinergic receptor P2Y2 (P2RY2) is a typical representative of G-protein coupled purinergic

receptors and widely distributed in normal tissues [9, 10]. P2RY2 is activated by UTP/ATP and participates in the regulation of various biological processes [10-13]. It has been reported P2RY2 is upregulated in certain cancers and promotes the proliferation of cancer cells including gastric[14], breast[13], pancreas[15], prostate[16] and lung[17]. Li and his colleagues [18] demonstrated that the corresponding enhancer of P2RY2 can transcribe into functional transcripts - P2RY2 enhancer RNA, termed P2RY2e after stimulus with estrogen. P2RY2e could act on chromatin looping to enhance gene transcription. Absence of P2RY2e led to reduced target gene expression and epigenetic features of P2RY2 enhancer, such as, histone modification and so on [19]. However, the roles of P2RY2e in BCa are completely 
ignorant. Given the persistence of gender differences in $\mathrm{BCa}$, estrogen appears to be particularly vital in the advancement of bladder cancer. It is also necessary to clarify whether P2RY2e involved in the effects of estrogen on BCa.

Our study explored the significance of P2RY2e and the potential molecular mechanisms of estrogen in tumorigenesis of $\mathrm{BCa}$. We first reported that P2RY2e was upregulated in BCa tissues and tightly associated with the histological grade, the depth of tumor infiltration and the TNM stage. We revealed estrogen could induce the production of P2RY2e and increase the malignant ability of BCa cells 5637 and T24. Knockdown of P2RY2e by CRISPR-Cas13a could inhibit cell multiplication, invasion and migration. Furthermore, the cell apoptosis was increased. And more importantly, the down-regulation of P2RY2e can reduce the cancer-promoting ability of estrogen on BCa cells.

\section{Materials and methods}

\subsection{Patient samples}

This research included a total of 38 BCa patients. $\mathrm{BCa}$ tissues and matched paracancerous tissues were quick-froze in liquid nitrogen after radical cystectomy. Written informed consent was obtained from all the patients. The study was approved by Institutional Review Board of Shenzhen Second People's Hospital.

\subsection{Cell culture and treatments}

Human BCa cell lines (5637, T24) were purchased from the America Type Culture Collection (ATCC, Manassas, VA, USA). The 5637 cells were cultured in phenol-free RPMI-1640 medium (Gibco, Thermo Fisher Scientific, Waltham, MA, USA) plus $10 \%$ charcoal-stripped fetal bovine serum (FBS) (Gibco). The T24 cells were cultured in phenol-free DMEM medium (Gibco, Thermo Fisher Scientific, Waltham, MA, USA) plus 10\% charcoal-stripped FBS (Gibco). All cells were placed in a humidified atmosphere of $5 \% \mathrm{CO} 2$ at $37^{\circ} \mathrm{C}$. Estrogen was used to stimulate the cells for $1 \mathrm{~h}$ to induce P2RY2e production.

\subsection{Cell transfection}

The small interfering RNA (siRNA) that targeted P2RY2e (si-P2RY2e) and negative control RNA (si-NC) were ordered from Syngen Tech Co., Beijing, China. The si-P2RY2e sequences were as follows: sense: 5'-GCAAAAGGUAGGAGGGUUU-3'; antisense: 5'- AAACCCUCCUACCUUUUGC-3' [18]. CRISPR-Cas13a targeting P2RY2e (Cas13a-P2RY2e) and negative control (Cas13a-NC) were constructed by Syngen Tech Co., Beijing, China. The guide RNA sequences of Cas13a-P2RY2e were as follows: ACTGTGAGGAGGCCCAGGCAGTACACCA. The guide RNA sequences of Cas13a-NC were as follows: TAGATTGCTGTTCTACCAAGTAATCCAT. BCa cells were seeded in 6-well plates and grown to a suitable density. Then cells were transfected with 100 pmol siRNA or $2.5 \mathrm{ug}$ plasmid vectors using Lipofectamine 3000 (Invitrogen, Carlsbad, CA, USA) according to the instructions.

\subsection{RNA extraction and RT-qPCR}

The total RNA of the tissue samples were extracted using TRIzol reagent (Invitrogen, Carlsbad, CA, USA) according to the manufacturer's instructions. 1ug total RNA was converted to cDNA using a Revertra Ace qPCR RT Kit (Toyobo, Osaka, Japan). GAPDH was used as the internal control for Real-time quantitative PCR (RT qPCR). The primer sequences were as follows: P2RY2e primers forward: 5'-AGGCAAATCCATTGTCATCC-3' and reverse: 5'-AACTGGCTGGATCTTGAAGC-3' [18]; GAPDH primers forward: 5'-AACGGATTTGGTCGTATTG-3' and reverse: 5'-GGAAGATGGTGATGGGATT-3'. RT qPCR was performed using ABI PRISM 7500 Fluorescent Quantitative PCR System (Applied Biosystems, Foster City, CA, USA). The reaction system included $5 \mathrm{ul}$ of qPCR Mix (Toyobo), $0.5 \mu \mathrm{l}$ of forward primer, $0.5 \mathrm{ul}$ of reverse primer, $1 \mathrm{ul}$ of cDNA and $3 \mathrm{ul}$ of deionized water. The $2-\Delta \Delta \mathrm{Ct}$ method was used to calculate the relative expression level. Each experiment was repeated at least three times.

\subsection{Cell proliferation assay}

Cell Counting Kit-8, CCK-8 (TransGen, Beijing, China) and EDU assay kit (RIBOBIO, Guangzhou, China) were used to examine cell proliferation. After $24 \mathrm{~h}$ of transfection, $5 \times 10^{3}$ cells were planted in 96-well plates. At 0, 24, 48 and 72h, 10ul CCK-8 and 90ul medium were incubated together at $37^{\circ} \mathrm{C}$ for $1 \mathrm{~h}$, then the absorbance at $450 \mathrm{~nm}$ was detected by a microplate reader (Bio-Rad, Hercules, CA, USA). For direct observation of the proliferating cells, 5-Ethynyl-2-deoxyuridine incorporation experiment was also performed according to the specifications. Each experiment was repeated at least three times. The mean in each condition is the average of the biological repeats.

\subsection{Cell motility assay}

Transwell assay was used to examine cell invasion. After Cas13a-P2RY2e or Cas13a-NC treatment for $24 \mathrm{~h}, 1.5 \times 10^{5} 5637$ cells and $5 \times 10^{4} \mathrm{~T} 24$ cells with $200 \mathrm{ul}$ serum-free medium were put into the upper chambers (24-well insert, pore size $8 \mu \mathrm{m}$,

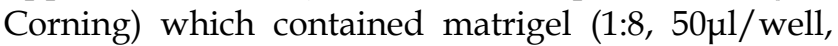
BD Bioscience, San Jose, CA, USA). The lower 
chambers were added to $500 \mu \mathrm{l}$ complete medium. After 24 hours of cultivation, the invaded cells were fixed with methanol for $20 \mathrm{~min}$, stained with $0.1 \%$ crystal violet for $25 \mathrm{~min}$ and photographed after drying. $1 \mathrm{ml} \mathrm{33 \%}$ acetic acid was added in each chamber with the invaded cells and the 24-well plates were soaked for $10 \mathrm{~min}$ to wash out the crystal violet. The absorbance at $570 \mathrm{~nm}$ was determined by a microplate reader (Bio-Rad, Hercules, CA, USA). Each experiment was performed at least three times. The mean in each condition is the average of the biological repeats.

Wound-healing assay was applied to assess cell migration. Clear lines were created with a sterile $200 \mu \mathrm{l}$ pipette tip after $24 \mathrm{~h}$ of transfection. Then the cells were continuously cultured in the medium supplemented with 1\% FBS for 24 hours. The wound areas were recorded at 0 hours and 24 hours using a digital camera system. The migration distance was measured by the software program ImageJ. Each experiment was repeated at least three times. The mean in each condition is the average of the biological repeats.

\subsection{Cell apoptosis assay}

We used caspase-3 ELISA assay and flow cytometry assay to detect cell apoptosis. BCa cells 5637 and T24 were transfected with Cas13a vectors in $10 \mathrm{~cm}$ dishes for $24 \mathrm{~h}$. Human caspase-3 ELISA assay kit (Cusabio, Wuhan, China) was used to detect the activity of caspase-3 according to the instructions. For flow cytometry assay, the cells were transfected with

a

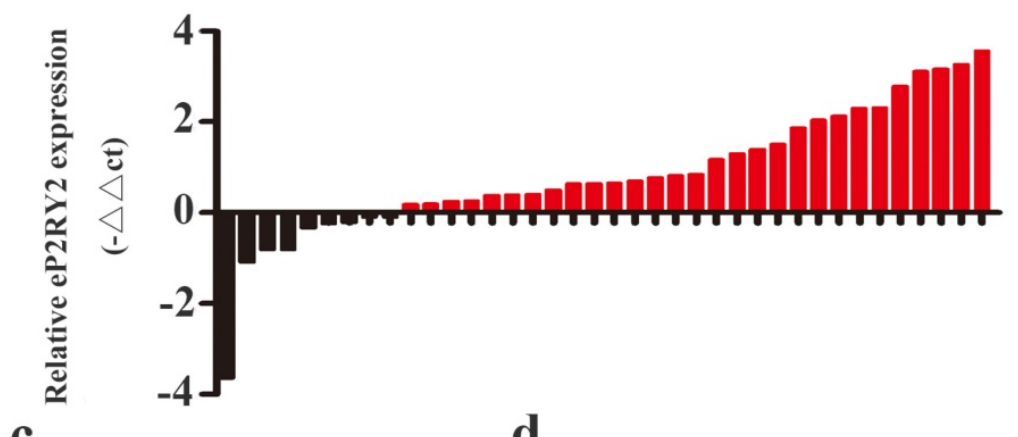

c

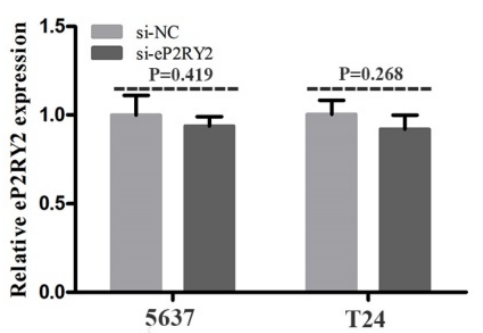

Cas13a vectors in 6-well plates for $24 \mathrm{~h}$. Then they were harvested and suspended with 100ul of Annexin $\mathrm{V}$ Binding Buffer. The cells were dyed with $5 \mathrm{ul}$ of Annexin V-FITC and 5ul of Propidium Iodide (PI) and incubated from light at room temperature. Cell apoptosis were analyzed using flow cytometry (EPICS, XL-4, Beckman, CA, USA). Each experiment was repeated at least three times.

\subsection{Statistical analysis}

All data were expressed as mean \pm standard deviation (SD) and all statistical analyses were performed by SPSS 17. Paired samples t-test was used to analyze the expression differences of P2RY2e between $\mathrm{BCa}$ tissues and matched normal tissues. The CCK-8 assay was analyzed using ANOVA. Other data was analyzed by the independent samples t-test. P < 0.05 was considered to be statistically significant.

\section{Results}

\subsection{P2RY2e was overexpressed in BCa and significantly associated with clinical pathological characteristics.}

We examined the relative expression levels of P2RY2e by RT qPCR in 38 BCa tissues together with adjacent tissues. Our results showed that P2RY2e was overexpression in 29 cancer tissues (Figure $1 \mathrm{a}$ and $1 \mathrm{~b}$ ). What's more, as shown in Table 1, increased P2RY2e expression was positively related to high histological grade, tumor invasion and the late TNM stage of bladder cancer. However, it didn't show a direct link

b

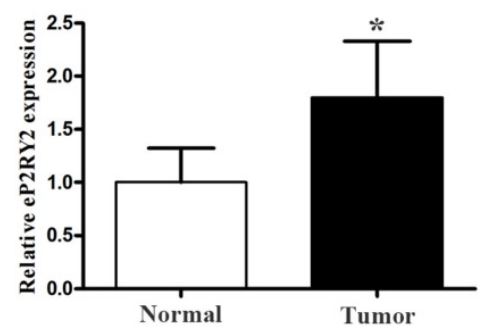

e
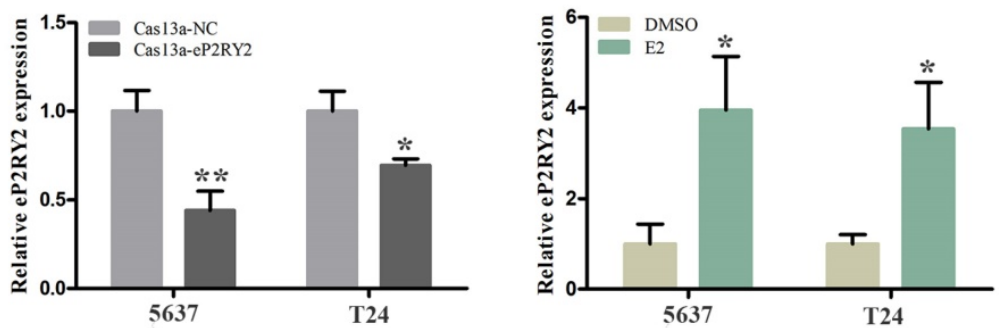

Figure 1: a, b The relative expression levels of P2RY2e were detected by RT-qPCR in a total of 38 BCa patients. P2RY2e was upregulated in BCa tissues compared to normal tissues. c siRNA can't effectively knock down P2RY2e. d P2RY2e expression was significantly downregulated by transfection of Cas 13a-P2RY2e. e P2RY2e expression was prominently increased in BCa cells after treatment with estrogen for 1 h. E2 represented estrogen. Data are shown as mean \pm SD. $\left({ }^{*} \mathrm{P}<0.05\right.$, ** $\left.\mathrm{P}<0.01\right)$ 
with the age, the gender and the lymph node metastasis. Accordingly, we concluded that P2RY2e played a cancer-promoting role in bladder cancer.

Table 1: Correlation between P2RY2e expression and clinicopathological characteristics of $B C a$ patients. $(* p<0.05$ was considered significant.)

\begin{tabular}{lllll}
\hline Characteristics & Total & \multicolumn{2}{l}{ P2RY2e expression } & P value \\
\hline \multicolumn{4}{l}{ Low } & High \\
Female & 7 & $2(28.6 \%)$ & $5(71.4 \%)$ & 0.852 \\
Male & 31 & $10(32.3 \%)$ & $21(67.7 \%)$ & \\
Age & & & & \\
$\leq 60$ & 10 & $3(30.0 \%)$ & $7(70.0 \%)$ & 0.425 \\
$>60$ & 28 & $5(17.9 \%)$ & $23(82.1 \%)$ & \\
Histological grade & & & & \\
Low & 15 & $9(60.0 \%)$ & $6(40.0 \%)$ & $0.018^{*}$ \\
High & 23 & $5(21.7 \%)$ & $18(78.3 \%)$ & \\
Depth of invasion (T) & & & & \\
Ta, T1 & 9 & $6(66.7 \%)$ & $3(33.3 \%)$ & $0.005^{* *}$ \\
T2, T3, T4 & 29 & $5(17.2 \%)$ & $24(82.8 \%)$ & \\
Lymph node metastasis & & & & \\
(N) & & & & \\
N0 & 36 & $6(16.7 \%)$ & $30(83.3 \%)$ & 0.243 \\
N1, N2, N3 & 2 & $1(50.0 \%)$ & $1(50.0 \%)$ & \\
TNM stage & & & & \\
$0 /$ I & 6 & $5(83.3 \%)$ & $1(16.7 \%)$ & $0.002^{* *}$ \\
II/III/IV & 32 & $6(18.8 \%)$ & $26(81.2 \%)$ & \\
\hline
\end{tabular}

\subsection{Targeting knockdown of P2RY2e with CRISPR-Cas 13 a was much more efficacious than siRNA.}

BCa cells 5637 and T24 were cultured and transfected with CRISPR-Cas13a vectors or siRNA. 24 hours later, we explored the relative expression of P2RY2e in BCa cells. Our results showed that siRNA failed to knock down P2RY2e effectively in 5637 and T24 (Figure 1c). In contrast, the relative expression of P2RY2e was decreased by $56.1 \%$ in 5637 and $26.3 \%$ in T24 (Figure 1d) by Cas13a-P2RY2e. These results indicated that only CRISPR-Cas13a was appropriate for the knockdown of P2RY2e.

\subsection{P2RY2e is upregulated in BCa cells when exposed to estrogen.}

We analyzed the P2RY2e expression level of $1 \mathrm{~h}$ estrogen-treated $(10 \mathrm{nM})$ BCa cells using RT qPCR. Our data revealed the relative expression level of P2RY2e was upregulated by 3.95-fold in 5637 and 3.54-fold in T24 after estrogen stimulation (Figure 1e). We demonstrated that $\mathrm{BCa}$ cells also responded to estrogen and then induced the synthesis of P2RY2e like breast cancer cells MCF-7.

\subsection{Estrogen promoted cell proliferation, invasion and migration, and inhibit cell apoptosis of BCa cells.}

To verify the impact of estrogen on BCa cells, we used both EDU assay and CCK- 8 assay to detect the proliferation of the cells with estrogen treatment. Compared with the control group, estrogen was able to stimulate cell growth of 5637 (Figure 2a, 2g, 3a) and T24 cells (Figure $2 \mathrm{~d}, 2 \mathrm{~g}, 3 \mathrm{a}$ ). Wound-healing assay and transwell assay were used to assess the cell motility of estrogen-treated cells. We found estrogen could significantly facilitate the cell invasion of 5637 and T24 cells (Figure 3d, 3g), as well as the cell migration (Figure 4a, 4d, 4g). Finally, we performed caspase-3 ELISA assay and flow cytometry assay to explore cell apoptosis. As expected, the activity of caspase- 3 protein in 5637 and T24 cells were decreased and the cell apoptosis was suppressed (Figure 5a, 5d, 5g). These results suggested the potential roles of estrogen in facilitating the initiation and invasion of $\mathrm{BCa}$.

\subsection{P2RY2e knockdown by specific Cas13a inhibit cell proliferation, invasion and migration, and induce cell apoptosis of $\mathrm{BCa}$ cells.}

We utilized CRISPR-Cas13a to explore the influence of P2RY2e downregulation on BCa cells. After Cas13a-P2RY2e or Cas13a-NC transfection for 24h, we studied changes in the malignant biological behaviors of BCa cells in the same way. Both EDU incorporation assay and CCK-8 assay exhibit prominent cell growth suppression of 5637 (Figure 2b, $2 \mathrm{~h}, 3 \mathrm{~b}$ ) and T24 cells (Figure 2e, 2h, 3b). The cell invasion were significantly decreased showed by transwell assay in 5637 and T24 (Figure 3e, 3h), as well as a weakened cell migration (Figure $4 \mathrm{~b}, 4 \mathrm{e}, 4 \mathrm{~h}$ ) showed by wound-healing assay. Besides, increased cell apoptosis was showed in 5637 and T24 by the detection of caspase-3 and flow cytometry assay (Figure 5b, 5e, 5h). Therefore, low expression of P2RY2e reduced the malignant ability of BCa cells.

\subsection{P2RY2e knockdown offset the carcinogenic effects of estrogen in $\mathrm{BCa}$ cells.}

In view of the P2RY2e induction of estrogen in 5637 and T24, we wondered P2RYe might participate in the carcinogenesis of estrogen on urinary bladder. We knocked down P2RY2e while adding estrogen and analyzed the malignant potential of BCa cells compared to the group treated with estrogen plus Cas3a-NC using the methods described above. We observed the cell proliferation (Figure 2c, 2f, 2i, 3c), invasion (Figure 3f, 3i) and migration (Figure 4c, 4f, 4i) induced by estrogen were weakened and the cell apoptosis was increased in case of P2RY2e knockdown (Figure 5c, 5f, 5i). In conclusion, P2RY2e downregulation can attenuate the acceerative effects of estrogen on BCa. 


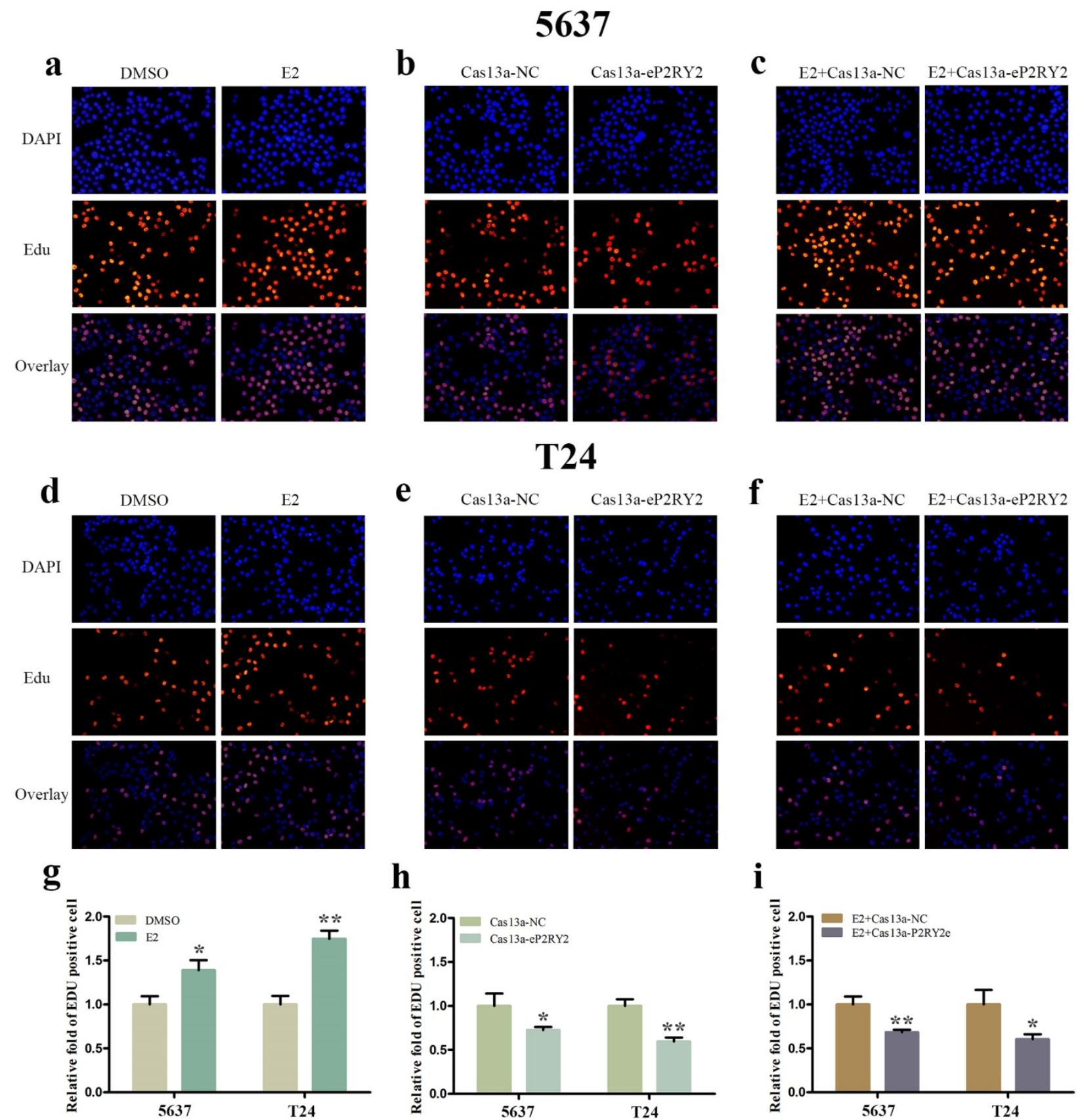

Figure 2: EDU assay was performed to investigate cell growth. a, d, $\mathbf{g}$ Estrogen promoted the multiplication of 5637 and T24 cells. b, e, $\mathbf{h}$ Downregulation of P2RY2e by Cas13a-P2RY2e inhibit cell proliferation of 5637 and T24 cells. c, f, i Downregulation of P2RY2e by Cas 13a-P2RY2e decreased cell proliferation induced by estrogen. E2 represented estrogen. Data are shown as mean \pm SD. $\left({ }^{*} p<0.05,{ }^{*} p<0.01\right)$

\section{Discussion}

$\mathrm{BCa}$ is the 9th most common malignancy worldwide, bringing a heavy economic burden to the world and poor quality of life to individuals [20,21]. The gender difference has forced us to think about the significance of estrogen in BCa. An exposition of more molecular mechanisms could deepen our understanding about the development of $\mathrm{BCa}$.

Enhancers are cis-acting elements which could regulate the transcriptional activation of target genes [22]. In recent years, several studies have proved that enhancers can transcribe into eRNAs when exposed to stimulus [18, 19, 23-25]. Increasing evidences demonstrate eRNAs are not just markers of activity enhancers, but play a functional role in gene transcription $[23,26,27] . \quad P 2 R Y 2 e$ is an estrogen-associated eRNA and could enhance gene transcription by facilitating gene looping [18, 19]. However, the roles of P2RY2e in BCa and the connection between P2RY2e and carcinogenesis of estrogen remain unclear. 
a
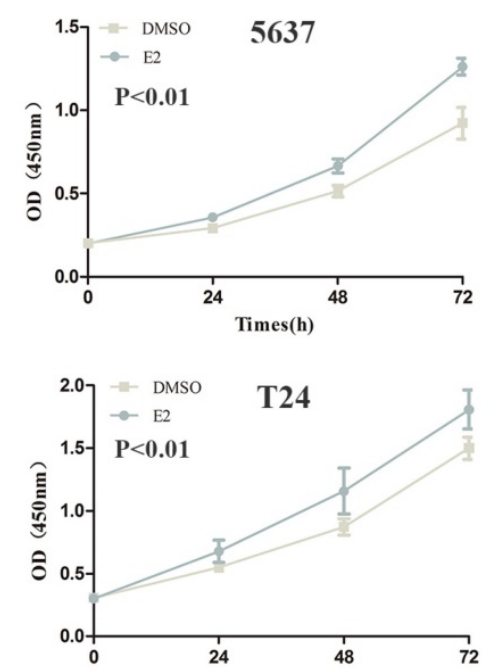

d
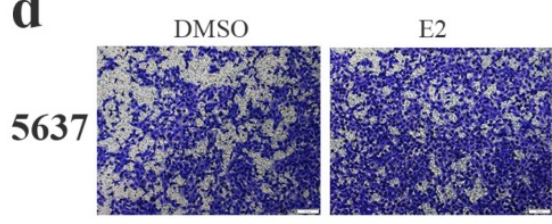

T24
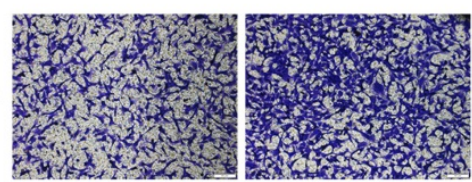

g

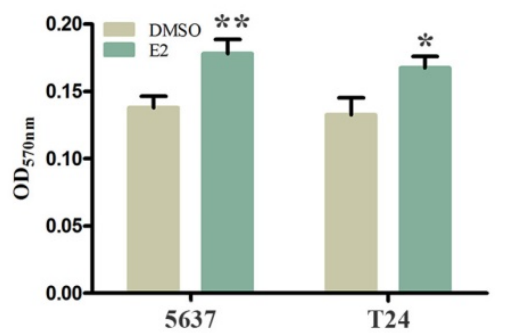

b
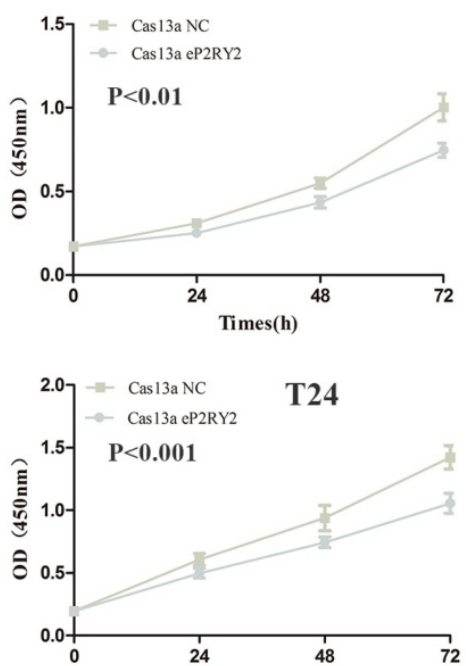

e
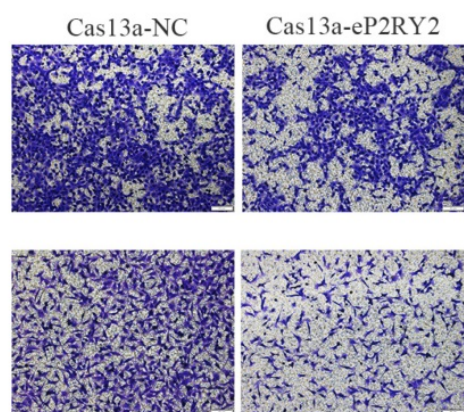

h

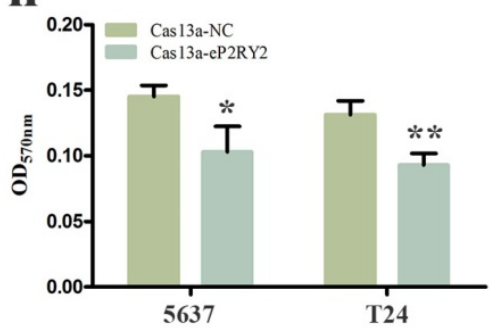

c
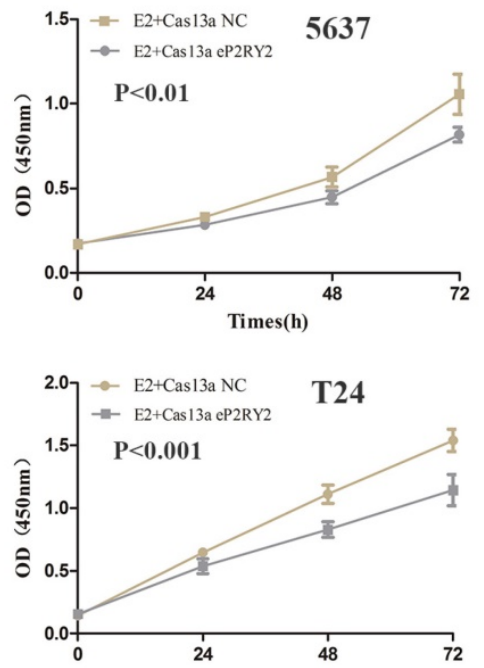

f
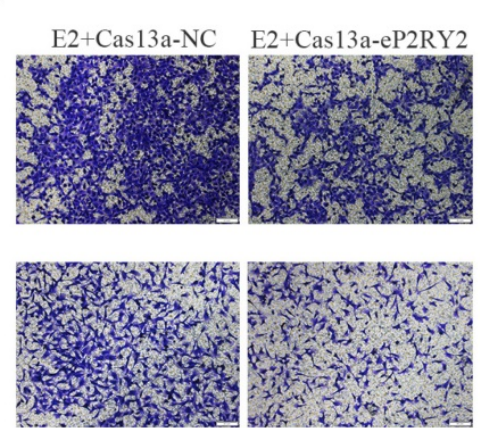

i

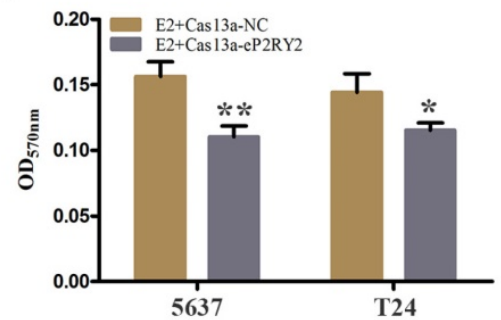

Figure 3: CCK-8 assay was used to detect cell proliferation as well. a Increased cell growth was observed in 5637 and T24 cells with stimulation of estrogen ( $10 \mathrm{mM}$ ). b Cell proliferation suppression was observed in 5637 and T24 cells transfected with Cas 13a-P2RY2e. c Estrogen-induced proliferation was attenuated after transfection with Cas 13a-P2RY2e in 5637 and T24. Transwell assay was used to detect cell invasion. d, $\mathbf{g}$ Estrogen promoted the cell invasion of 5637 and T24 cells. e, $\mathbf{h}$ Cell invasion was inhibit in 5637 and T24 after transfection of Cas13a-P2RY2e. f, i Compared with the negative control, cell invasion was inhibit with estrogen treatment and Cas13a-P2RY2e transfection. E2 represented estrogen. Data are shown as mean \pm SD. $\left({ }^{*} p<0.05, * * p<0.01, * * * p<0.001\right)$

Our study demonstrated that P2RY2e is highly expressed in BCa tissues and closely associated with the histological grade, the depth of invasion and the clinical TNM stage for the first time. Possibly due to limited numbers of tumor samples, we did not get any significant connections among gender, age, lymph node metastasis and BCa. In summary, our results showed P2RY2e may serve as a driver in the occurrence and progression of $\mathrm{BCa}$.

We knocked down P2RY2e for further investigation in $\mathrm{BCa}$. It may be difficult to access to the nucleus, transfection of siRNA provided us an extremely poor knockdown efficiency of P2RY2e. Next, we designed a guide RNA specifically targeting P2RY2e and constructed P2RY2e silencing vectors by LwaCas13a reported by Zhang et al. which was different from other CRISPR systems and had the ability to knock down the nuclear transcripts precisely and efficiently based on the nuclear localization sequence[28]. Our results showed the knockdown efficiency of CRISPR-Cas13a is much better than that of siRNA. So we believed that CRISPR-Cas13a was much more applicable to the knockdown of P2RY2e. 

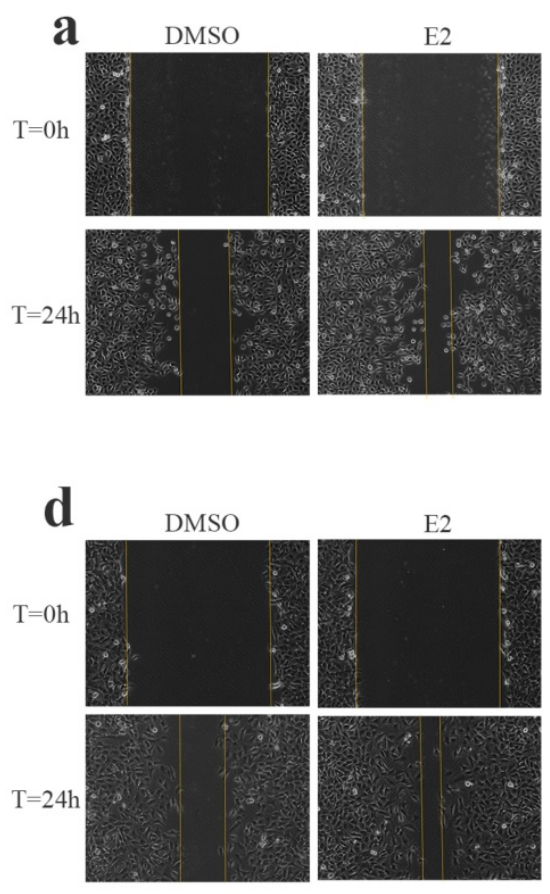

g

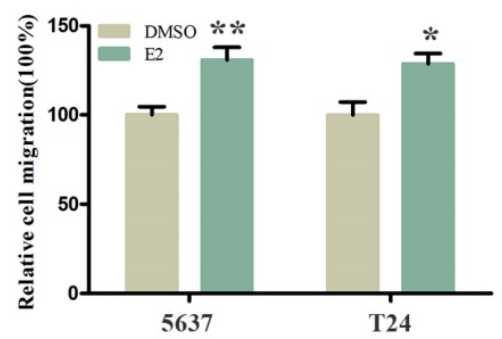

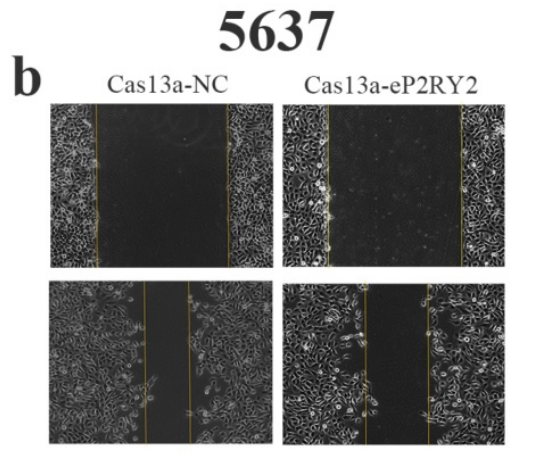
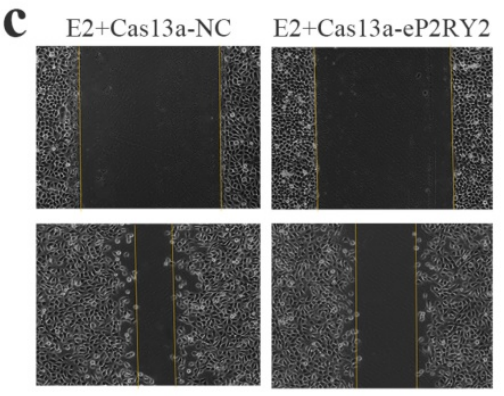

\section{T24}

e

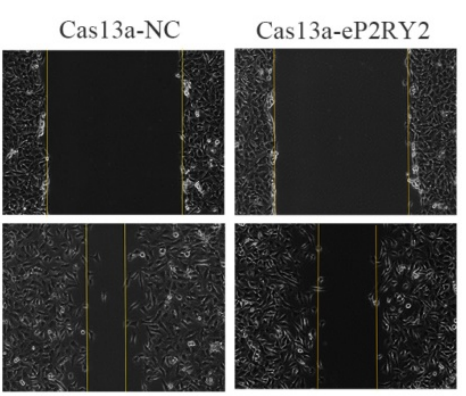

h

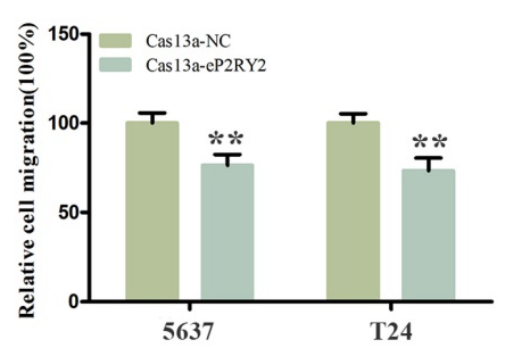

f

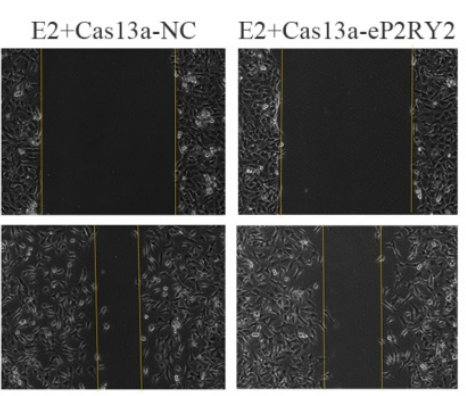

i

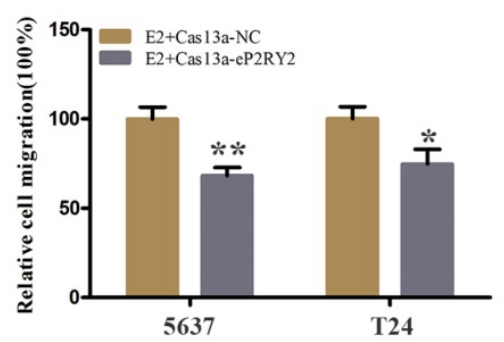

Figure 4: Cell migration was analyzed by wound-healing assay. a, d, g Estrogen promoted the cell migration of 5637 and T24 cells. b, e, h After transfection of Cas 13a-P2RY2e, cell migration was inhibit in 5637 and T24. c, f, i Cell migration was inhibit with estrogen treatment and Cas13a-P2RY2e transfection compared to the negative control. E2 represented estrogen. Data are shown as mean \pm SD. $\left({ }^{*} \mathrm{p}<0.05\right.$, $\left.{ }^{* *} \mathrm{p}<0.01\right)$

To further clarify the roles of P2RY2e in $\mathrm{BCa}$, we firstly studied the effects of P2RY2e downregulation on BCa cells. Inhibit malignant abilities of the cells were observed, which was suggested that P2RY2e might play functional roles in promoting BCa. We discovered that estrogen could facilitate the malignant biological behaviors of BCa cells, as well as inducing the synthesis of P2RY2e. We considered there might be a unique connection between P2RY2e and cancer-promoting of estrogen. So we explored whether estrogen promoted the malignant potential of BCa cells when P2RY2e was knocked down. As expected we observed the cell proliferation, invasion and migration induced by estrogen were impaired and cell apoptosis were increased after Cas13a-P2RY2e transfection. There have been reported that the estrogen receptor $\beta$ (ER $\beta$ ) plays a major role in the proliferation of cells[29]. Whether the P2RY2e work in conjunction with ER $\beta$ to induce malignant behaviors of $\mathrm{BCa}$ cells need a thorough research. As a conclusion, we determined that P2RY2e undoubtedly participated in the carcinogenesis of estrogen on bladder cancer.

\section{Conclusion}

For the first time, we revealed P2RY2e was overexpressed in $\mathrm{BCa}$ tissues compared to the matched normal tissues. Downregulation of P2RY2e could suppress the malignant phenotype of BCa. Our results have revealed that $P 2 R Y 2 e$ acts as a carcinogenic factor in BCa. What's more, it is generally recognized that there is a close relationship between estrogen and $\mathrm{BCa}$, but the molecular mechanism of estrogen action in $\mathrm{BCa}$ remains puzzled. We have demonstrated that estrogen may promote the initiation and invasion of $\mathrm{BCa}$ by the induction of P2RY2e. In conclusion, our study provides a new outlook and a fresh perspective on the molecular mechanisms of sex hormones acting on $\mathrm{BCa}$. We have revealed that enhancer RNAs could act 
a

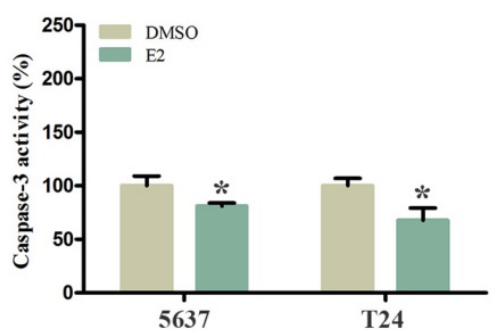

d
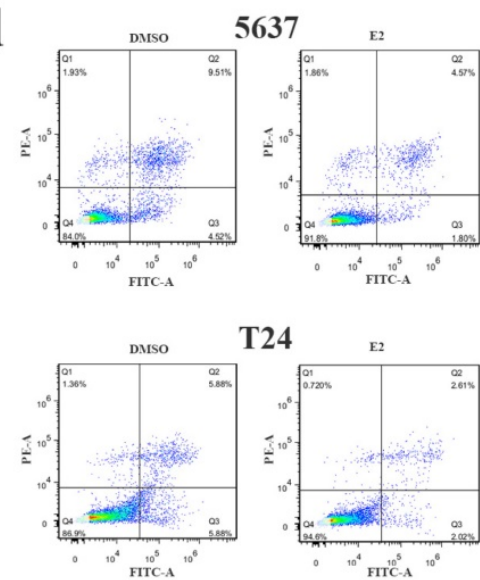

T24

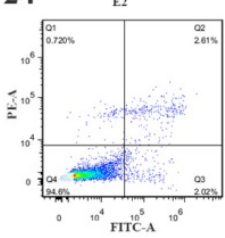

g

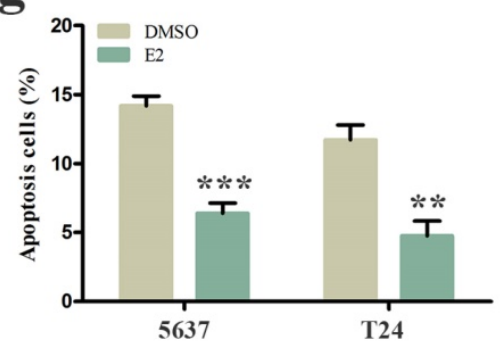

b

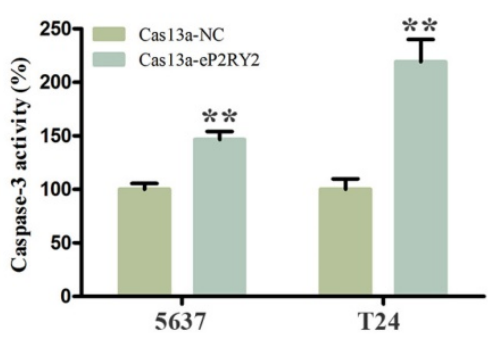

e

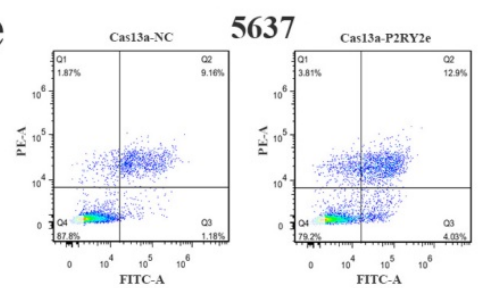

T24
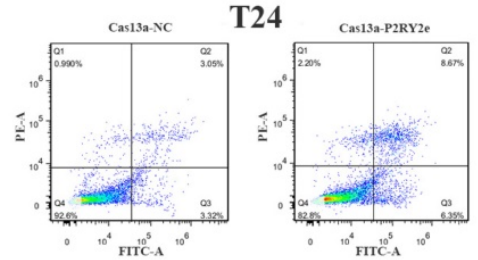

h

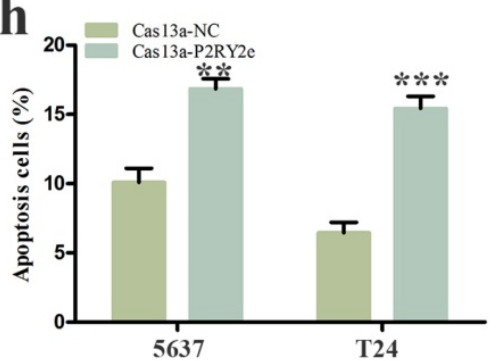

c

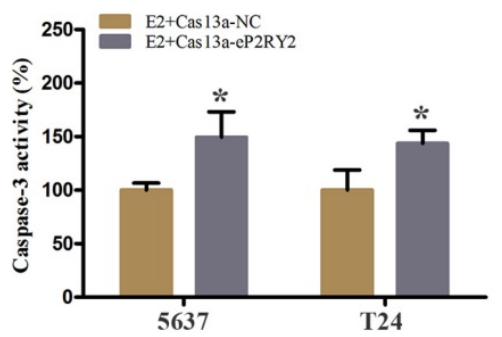

f
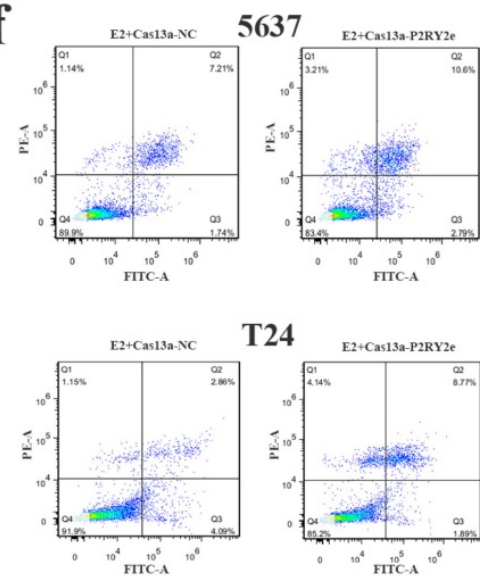

T24

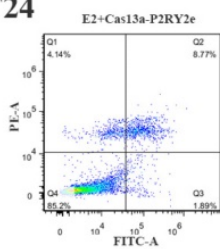

i

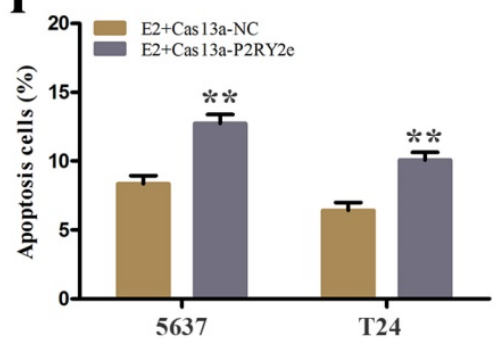

Figure 5: Cell apoptosis was measured by caspase-3 ELISA assay and flow cytometry assay. a, d. g Estrogen decreased the cell apoptosis of 5637 and T24 cells. b, e, h P2RY2e downregulation increased cell apoptosis of 5637 and T24 cells. c, f, i Cell apoptosis was facilitated with estrogen treatment and Cas 13 a-P2RY2e transfection compared to the negative control. E2 represented estrogen. Data are shown as mean \pm SD. $\left({ }^{*} p<0.05, * * p<0.01, * * * p<0.001\right)$

as a potential target for clinical therapeutics of BCa. More effective treatment strategies targeting P2RY2e of BCa need to be developed in future research.

\section{Abbreviations}

enhancer RNAs: eRNAs; P2RY2 enhancer RNA: P2RY2e; Standard deviation: SD; Bacillus Calmette-Guerin: BCG; Purinergic receptor P2Y2: P2RY2; Histone 3 Lysine 4 monomethylation: H3K4me1; Histone 3 lysine 27 acetylation: H3K27ac; Small interfering RNA: siRNA; Real-time quantitative PCR: RT qPCR; Cell Counting Kit-8: CCK-8; Propidium Iodide: PI.

\section{Acknowledgments}

The authors are particularly grateful to all the patients participated in our study.

\section{Ethics approval and consent to participate}

The study was approved by the Institutional Research Ethics Committee of Shenzhen Second People's Hospital. All the patients signed a written informed consent.

\section{Funding}

This work was supported by the National Key Basic Research Program of China (973 Program) (2014CB745201), National Natural Science Foundation of China (81772737), the Shenzhen Municipal Government of China (JCYJ20170413161749433, JSGG20160301161836370), the Sanming Project of Shenzhen Health and Family Planning Commission, SZSM201412018, SZSM201512037. The high level university's medical discipline construction 2016031638. 


\section{Competing Interests}

The authors have declared that no competing interest exists.

\section{References}

1. Horie S. Editorial comment to Inverse expression of estrogen receptor-beta and nuclear factor-kappaB in urinary bladder carcinogenesis. International journal of urology : official journal of the Japanese Urological Association. 2010; 17: 810.

2. Bolenz C, Lotan Y, Ashfaq R, Shariat SF. Estrogen and progesterone hormonal receptor expression in urothelial carcinoma of the bladder. European urology. 2009; 56: 1093-5.

3. Xu Y, Zhang NZ, Chen J, Yuan HQ. Biomarkers in urothelial carcinoma of the bladder: the potential cross-talk between transforming growth factor-beta1 and estrogen receptor beta/androgen receptor pathways. Medical hypotheses. 2013; 80: 716-8.

4. Madeb R, Messing EM. Gender, racial and age differences in bladder cancer incidence and mortality. Urologic oncology. 2004; 22: 86-92.

5. Murta-Nascimento C, Schmitz-Drager BJ, Zeegers MP, Steineck G, Kogevinas M, Real FX, et al. Epidemiology of urinary bladder cancer: from tumor development to patient's death. World journal of urology. 2007; 25: 285-95.

6. Hartge P, Harvey EB, Linehan WM, Silverman DT, Sullivan JW, Hoover RN, et al. Unexplained excess risk of bladder cancer in men. Journal of the National Cancer Institute. 1990; 82: 1636-40.

7. Racioppi M, D'Agostino D, Totaro A, Pinto F, Sacco E, D'Addessi A, et al. Value of current chemotherapy and surgery in advanced and metastatic bladder cancer. Urologia internationalis. 2012; 88: 249-58.

8. Rose TL, Milowsky MI. Improving Systemic Chemotherapy for Bladder Cancer. Current oncology reports. 2016; 18: 27.

9. Faria R, Ferreira L, Bezerra R, Frutuoso V, Alves L. Action of natural products on p2 receptors: a reinvented era for drug discovery. Molecules. 2012; 17: 13009-25.

10. von Kugelgen I, Harden TK. Molecular pharmacology, physiology, and structure of the P2Y receptors. Advances in pharmacology. 2011; 61: 373-415.

11. Martinez-Ramirez AS, Garay E, Garcia-Carranca A, Vazquez-Cuevas FG. The P2RY2 Receptor Induces Carcinoma Cell Migration and EMT Through Cross-Talk With Epidermal Growth Factor Receptor. Journal of cellular biochemistry. 2016; 117: 1016-26.

12. Jacobson KA, Paoletta S, Katritch V, Wu B, Gao ZG, Zhao Q et al. Nucleotides Acting at P2Y Receptors: Connecting Structure and Function. Molecular pharmacology. 2015; 88: 220-30.

13. Li HJ, Wang LY, Qu HN, Yu LH, Burnstock G, Ni X, et al. P2Y2 receptor-mediated modulation of estrogen-induced proliferation of breast cancer cells. Molecular and cellular endocrinology. 2011; 338: 28-37.

14. Aquea G, Bresky G, Lancellotti D, Madariaga JA, Zaffiri V, Urzua U, et al. Increased expression of P2RY2, CD248 and EphB1 in gastric cancers from Chilean patients. Asian Pacific journal of cancer prevention : APJCP. 2014; 15: 1931-6.

15. Choi JH, Ji YG, Lee DH. Uridine triphosphate increases proliferation of human cancerous pancreatic duct epithelial cells by activating P2Y2 receptor. Pancreas. 2013; 42: 680-6.

16. Li WH, Qiu Y, Zhang HQ, Liu Y, You JF, Tian XX, et al. P2Y2 receptor promotes cell invasion and metastasis in prostate cancer cells. British journal of cancer. 2013; 109: 1666-75.

17. Schumacher D, Strilic B, Sivaraj KK, Wettschureck N, Offermanns S. Platelet-derived nucleotides promote tumor-cell transendothelial migration and metastasis via P2Y2 receptor. Cancer cell. 2013; 24: 130-7.

18. Li W, Notani D, Ma Q, Tanasa B, Nunez E, Chen AY, et al. Functional roles of enhancer RNAs for oestrogen-dependent transcriptional activation. Nature. 2013; 498: 516-20.

19. Hah N, Murakami S, Nagari A, Danko CG, Kraus WL. Enhancer transcripts mark active estrogen receptor binding sites. Genome research. 2013; 23: 1210-23.

20. Ferlay J, Soerjomataram I, Dikshit R, Eser S, Mathers C, Rebelo M, et al. Cancer incidence and mortality worldwide: sources, methods and major patterns in GLOBOCAN 2012. International journal of cancer. 2015; 136: E359-86.

21. Breyer J, Wirtz RM, Laible M, Schlombs K, Erben P, Kriegmair MC, et al. ESR1, ERBB2, and Ki67 mRNA expression predicts stage and grade of non-muscle-invasive bladder carcinoma (NMIBC). Virchows Archiv : an international journal of pathology. 2016; 469: 547-52.

22. Lee K, Hsiung CC, Huang P, Raj A, Blobel GA. Dynamic enhancer-gene body contacts during transcription elongation. Genes \& development. 2015; 29: 1992-7.

23. Hsieh $\mathrm{CL}$, Fei $\mathrm{T}$, Chen $\mathrm{Y}, \mathrm{Li} \mathrm{T}$, Gao $\mathrm{Y}$, Wang $\mathrm{X}$, et al. Enhancer RNAs participate in androgen receptor-driven looping that selectively enhances gene activation. Proceedings of the National Academy of Sciences of the United States of America. 2014; 111: 7319-24.

24. Nagarajan S, Hossan T, Alawi M, Najafova Z, Indenbirken D, Bedi U, et al. Bromodomain protein BRD4 is required for estrogen receptor-dependent enhancer activation and gene transcription. Cell reports. 2014; 8: 460-9.
25. Pnueli L, Rudnizky S, Yosefzon Y, Melamed P. RNA transcribed from a distal enhancer is required for activating the chromatin at the promoter of the gonadotropin alpha-subunit gene. Proceedings of the National Academy of Sciences of the United States of America. 2015; 112: 4369-74.

26. Lam MT, Cho H, Lesch HP, Gosselin D, Heinz S, Tanaka-Oishi Y, et al. Rev-Erbs repress macrophage gene expression by inhibiting enhancer-directed transcription. Nature. 2013; 498: 511-5.

27. Kim TK, Hemberg M, Gray JM, Costa AM, Bear DM, Wu J, et al. Widespread transcription at neuronal activity-regulated enhancers. Nature. 2010; 465: $182-7$

28. Abudayyeh OO, Gootenberg JS, Essletzbichler P, Han S, Joung J, Belanto JJ, et al. RNA targeting with CRISPR-Cas13. Nature. 2017; 550: 280-4.

29. Kauffman EC, Robinson BD, Downes M, Marcinkiewicz K, Vourganti S, Scherr DS, et al. Estrogen receptor-beta expression and pharmacological targeting in bladder cancer. Oncol Rep. 2013; 30: 131-8. 